\title{
Primeiro registro de Psychodopygus lloydi (Antunes) (Diptera, Psychodidae) no Estado do Paraná, Sul do Brasil
}

\author{
Demilson Rodrigues dos Santos ${ }^{1}$, Ademar Rodrigues dos Santos ${ }^{1}$, Otílio de Oliveira ${ }^{1}$, Luiz Paschoal Poiani ${ }^{1}$ \\ \& Allan Martins da Silva ${ }^{2}$
}

\begin{abstract}
${ }^{1}$ Laboratório de Entomologia Médica, Secretaria de Estado da Saúde do Paraná, Travessa Jefferson Wanderley 718, 87.045-110, Vila NovaMaringá-PR, Brasil. demilson.entomologia@ig.com.br

${ }^{2}$ Coordenadoria de Pesquisas em Entomologia Médica, Secretaria de Estado da Saúde do Paraná, Rua Piquiri 170, 80.230-140, Curitiba-PR allanms@ibest.com.br
\end{abstract}

\begin{abstract}
First report of Psychodopygus lloydi (Antunes) (Diptera, Psychodidae) in Paraná State, southern of Brazil. This work reports the presence of Psychodopygus lloydi in Paraná State, South Region of Brazil, near the border of Santa Catarina State. The specimens were captured in the Municipality of São Mateus do Sul, using Falcão light trap in banks or inside the forest, on the soil and in the canopy level, respectively, between 6 p.m and 6 a.m. This investigation contributes to the advance of the studies on the geographical distribution of Phlebotomine sand fly species in Brazil.
\end{abstract}

KEYWORDS. Geographical distribution; sand flies; Phlebotominae.

\begin{abstract}
RESUMO. Primeiro registro de Psychodopygus lloydi (Antunes) (Diptera, Psychodidae) no Estado do Paraná, Sul do Brasil. O presente trabalho relata pela primeira vez a coleta de Psychodopygus lloydi no Estado do Paraná, Região Sul do Brasil, na divisa com o Estado de Santa Catarina. Os espécimes foram capturados no Município de São Mateus do Sul, usando armadilhas de Falcão, na margem e no interior de mata, em nível de solo e copa, respectivamente, entre as 18 e 6 horas. O resultado deste trabalho contribui para o avanço do conhecimento da distribuição geográfica dos flebotomíneos no Brasil.
\end{abstract}

PALAVRAS-CHAVE. Distribuição geográfica; flebotomíneos; Phlebotominae.

Psychodopygus lloydi foi descrito por Antunes (1937) a partir de fêmeas capturadas no Município de São Paulo, Estado de São Paulo. Em seguida, Coutinho (1940) descreveu o macho desta espécie, com base em exemplares coletados em Mogi das Cruzes, igualmente no Estado de São Paulo. A associação errônea com os machos de Psychodopygus arthuri Fonseca (1939) foi corrigida por Galati (1981).

Até o presente momento, ao que tudo indica, parece que Psychodopygus lloydi é uma espécie com distribuição regional. Consta que a distribuição geográfica da referida espécie encontra-se restrita às Regiões Sudeste e Centro-Oeste do Brasil, representadas pelos Estados do Rio de Janeiro no município de Petrópolis, São Paulo nos municípios de Osasco, Piedade e São Paulo, e com maior abrangência no Estado de Minas Gerais nos municípios de Belo Horizonte, Belo Vale, Caeté, Conceição do Mato Dentro, Entre Rios de Minas, Itabira, Nova Lima, Oliveira, Ouro Preto, Pirapora, Pitangui, Sabará e Santa Bárbara (Andrade Filho et al. 1997). Foi também coletado no Estado do Mato Grosso do Sul, sem referência aos municípios (Aguiar \& Medeiros 2003).

Neste trabalho registra-se pela primeira vez este flebotomíneo na Região Sul, mais precisamente no Município de São Mateus do Sul, Mesorregião Sudeste do Estado do Paraná, situado na divisa com o Estado de Santa Catarina.

Distando cerca de $150 \mathrm{~km}$ da Capital (Curitiba), o município de São Mateus do Sul encontra-se no segundo planalto paranaense, numa altitude aproximada de $800 \mathrm{~m}, 25^{\circ} 52^{\prime} 26^{\prime \prime} \mathrm{S}$ e $50^{\circ} 22^{\prime} 58^{\prime \prime} \mathrm{W}$. O clima da região é do tipo Cfb de Köppen, subtropical úmido sem estação seca, precipitação média anual entre 1400 e $1500 \mathrm{~mm}$, com temperatura média do mês mais quente inferior a $22^{\circ} \mathrm{C}$ e a média do mês mais frio superior a $10^{\circ} \mathrm{C}$, com mais de cinco geadas por ano.

Os flebotomíneos foram coletados na área rural (Sítio do Sr. Márcio), cerca de $10 \mathrm{~km}$ da área urbana, na direção Norte. As coletas foram realizadas no período noturno, nos dias 3/8/ 2005 e 6, 7, 8 e 9/2/2006. Utilizou-se armadilhas elétricas luminosas do tipo Falcão, instaladas em residências, abrigos de animais domésticos (chiqueiros e galinheiros), em buracos de tatu, na margem e no interior de mata do tipo Floresta Subtropical, composta de árvores de grande e pequeno porte, em nível de solo e copa, respectivamente, a 1 e 15 metros de altura, entre as 18 e 6 horas do dia seguinte. Também foram realizadas coletas simultâneas com armadilha de Shannon no interior de mata, porém das 18 às 21 horas. 
O material coletado foi identificado mediante chave para identificação específica, segundo Galati (2003b) e encontra-se depositado na Coleção Entomológica do Laboratório de Entomologia Médica de Maringá, PR. A classificação das espécies nos gêneros foi feita de acordo com a proposta de Galati (2003a).

Foi obtido, com as duas técnicas de captura, um total de 185 exemplares de flebotomíneos, dentre os quais foram identificadas três fêmeas de Psychodopygus lloydi, sendo duas capturadas na margem de mata (solo) e uma no interior de mata (copa), ambas em armadilhas de Falcão, respectivamente, nos dias 3/8/2005 e 9/2/2006. O restante do material era constituído por Brumptomyia troglodytes (Lutz, 1922), Migonemyia migonei (França, 1920), Pintomyia fischeri (Pinto, 1926), Pintomyia monticola (Costa Lima, 1932) e Psathyromyia shannoni (Dyar, 1929).

A maioria das espécies do gênero Psychodopygus possui hábitos exclusivamente silvestres. Segundo Rangel \& Lainson (2003), Psychodopygus wellcomei (Fraiha, Shaw \& Lainson, 1971), Psychodopygus complexa (Mangabeira, 1941), Psychodopygus paraensis (Costa Lima, 1941) e Psychodopygus ayrozai (Barretto \& Coutinho, 1940) são consideradas importantes na transmissão de Leishmaniose Tegumentar Americana, sobretudo nas Regiões Norte e Nordeste.

Marcondes et al. (2001) ao estudar a fauna flebotomínea em uma reserva de mata primária do município de Morretes, Região Leste do Estado do Paraná, detectaram a presença de nove espécies de flebotomíneos; dentre elas, Ps. ayrozai e Psychodopygus geniculata (Mangabeira, 1941), foram as mais abundantes. Neste mesmo trabalho, Ps. ayrozai é referida como sendo uma espécie comum em floresta secundária de Florianópolis, Estado de Santa Catarina.

Embora, até o presente momento não se tenha nenhuma informação sobre o envolvimento de Ps. lloydi na transmissão de Leishmania, cabe destacar a importância deste relato sobre seu encontro no extremo sul do Estado do Paraná, onde as médias de temperaturas costumam ser mais amenas em relação às das regiões onde a espécie ocorre (Andrade Filho et al.
1997; Aguiar \& Medeiros 2003). Em suma, o resultado deste trabalho contribui com os avanços dos estudos de distribuição geográfica dos flebotomíneos no Brasil.

Agradecimentos. À Dra. Eunice Aparecida Bianchi Galati, pela confirmação das espécies de flebotomíneos.

\section{REFERÊNCIAS}

Aguiar, G. M. \& W. M. Medeiros. 2003. Distribuição regional de habitats das espécies de flebotomíneos do Brasil. pp. 207-256. In: Rangel E. F. \& R. Laison (Org.) Flebotomíneos do Brasil. Rio de Janeiro: Fiocruz, iii+367 p.

Andrade Filho, J. D.; R. P. Brazil \& A. L. Falcão. 1997. Nota sobre a distribuição geográfica de Lutzomyia (Psychodopygus) arthuri (Fonseca) e Lutzomyia (Psychodopygus) lloydi (Antunes) (Diptera: Psychodidae). Anais da Sociedade Entomológica do Brasil 26: 403-405.

Antunes, P. C. A. 1937. Nota sobre Flebotomus Sul-americanos. I. Um novo Flebotomus, Flebotomus lloydi, encontrado em São Paulo (Diptera, Psychodidae). Revista de Biologia e Hygiene 8: 2426.

Coutinho, J. O. 1940. Observações sobre algumas espécies de Flebotomus com a descrição do macho de Flebotomus lloydi Antunes, 1937. Arquivos de Zoologia do Estado de São Paulo 1: $331-336$.

Galati, E. A. B. 1981. Sobre a identificação de Psychodopygus (Psychodopygus) arthuri (Fonseca, 1936) e P. (P.) lloydi (Antunes, 1937) (Diptera, Psychodidae, Phlebotominae). Revista Brasileira de Entomologia 25: 321-322.

Galati, E. A. B. 2003a. Classificação de Phlebotominae. pp. 23-51 In: Rangel E. F. \& R. Lainson (Org.). Flebotomíneos do Brasil. Rio de Janeiro: Fiocruz, ii +367 p.

Galati, E. A. B. 2003b. Morfologia, terminologia de adultos e identificação dos táxons da América. pp. 53-175. In: Rangel E. F. \& R. Lainson (Org.). Flebotomíneos do Brasil. Rio de Janeiro: Fiocruz, ii+367 p.

Marcondes, C. B.; Santos-Neto L. G. \& A. L. Lozovei. 2001. Ecology of Phlebotomine sandflies (Diptera, Psychodidae) in Brazilian Atlantic Forest. Revista da Sociedade Brasileira de Medicina Tropical 34: 225-260.

Rangel, E. F. \& R. Lainson. 2003. Transmissores de Leishmaniose Tegumentar Americana. pp. 291-310. In: Rangel E. F. \& R. Lainson (Org.). Flebotomíneos do Brasil. Rio de Janeiro: Fiocruz, vi+ $367 \mathrm{p}$. 